\title{
Performance Analysis of the Dual-Hop Asymmetric Fading Channel
}

\author{
Himal A. Suraweera, Member, IEEE, George K. Karagiannidis, Senior Member, IEEE, \\ and Peter J. Smith, Senior Member, IEEE
}

\begin{abstract}
In real wireless communication environments, it is highly likely that different channels associated with a relay network could experience different fading phenomena. In this paper, we investigate the end-to-end performance of a dualhop fixed gain relaying system when the source-relay and the relay-destination channels experience Rayleigh/Rician and Rician/Rayleigh fading scenarios respectively. Analytical expressions for the cumulative distribution function of the end-to-end signal-to-noise ratio are derived and used to evaluate the outage probability and the average bit error probability of $M$-QAM modulations. Numerical and simulation results are presented to illustrate the impact of the Rician factor on the end-toend performance. Furthermore, these results confirm that the system exhibits an improved performance in a Rician/Rayleigh (source-relay link/relay-destination link) environment compared to a Rayleigh/Rician environment.
\end{abstract}

Index Terms-Wireless relays, amplify-and-forward, Rayleigh fading, Rician Fading, outage probability, average bit error probability.

\section{INTRODUCTION}

$\mathbf{R}$ ECENTLY, wireless relaying techniques have become a major topic in the wireless research community due to their possible application in cellular, ad-hoc networks and military communications [1]. Amplify-and-Forward (AF) relaying is a popular method for implementing relay systems. Compared to Decode-and-Forward (DF) which employs full decoding and re-encoding at the relay, AF requires less processing power at the relay [2]. An AF relay amplifies the received signal from the source and then retransmits towards the destination [3]. Therefore, at the relay no decoding is performed. AF relays can be broadly categorized as: 1) channel state information (CSI) assisted relays, and 2) fixed gain or "blind" relays [4]. Fixed gain relays use power amplifiers with a constant gain to amplify the signals and exhibit a performance close to that of the systems with (CSI)-assisted relays [3].

Several works have analysed the performance of AF relay networks (operating under different fading conditions) using

Manuscript received March 27, 2008; revised July 25, 2008, October 28, 2008, and December 17, 2008; accepted January 27, 2009. The associate editor coordinating the review of this letter and approving it for publication was S. Ghassemzadeh.

This work was supported by a Discovery Grant (DP0774689) from the Australian Research Council.

H. A. Suraweera is with the Center for Telecommunications and Microelectronics, Victoria University, PO Box 14428, Melbourne, Victoria 8001, Australia (e-mail: himal.suraweera@vu.edu.au).

G. K. Karagiannidis is with the Department of Electrical and Computer Engineering, Aristotle University of Thessaloniki, GR-54124, Greece (e-mail: geokarag@auth.gr).

P. J. Smith is with the Department of Electrical and Computer Engineering, The University of Canterbury, Private Bag 4800, Christchurch, New Zealand (e-mail: p.smith@elec.canterbury.ac.nz).

Digital Object Identifier 10.1109/TWC.2009.080420 the outage probability of the end-to-end signal-to-noise ratio (SNR) and the average bit error probability (ABEP). (See [3]-[7] and references therein). In [3], Hasna and Alouini have studied the ABEP of dual-hop systems with AF relaying over Rayleigh fading channels. In [5] and [6], Karagiannidis et al. have studied the performance bounds of AF multihop transmissions over non-identically distributed Nakagami- $m$ fading channels. More recently, in [4], Mheidat and Uysal have investigated the impact of receive diversity on the performance of a relay-assisted network in which the relay is operating under the fixed gain constraint. Zhao et al. [7] presented an asymptotic SER study of a selection AF network where the "best" relay which contributes most to received SNR is chosen for retransmission.

In addition to the widely used Rayleigh and Nakagami- $m$ fading assumptions, Rician fading is often used in the technical literature to model wireless propagation comprising with a line-of-sight (LoS) component and a scattered component [8]. A recently released WINNER II project deliverable [9] also documents Rician propagation characteristics in micro/macro cellular multi-hop transmissions. Despite the importance of the Rician model, only a few works have analyzed the performance of relays under LoS fading conditions [10], [11]. For example, the performance of Decode-and-Forward (DF) relay networks under Rician fading has been considered in [10]. The symbol error probability of a multiple-input multiple-output semi-blind relay system has been studied in [11].

Recently, some papers have also studied the performance of relay networks under asymmetric fading scenarios [12], [13]. Asymmetric fading conditions, where links associated in the relay network are subject to different fading distributions, may arise due to the close proximity of the terminals or shadowing effects. In [12], Adinoyi and Yanikomeroglu have studied the error performance for a DF relay network by considering different signal strengths/severities such as non-LoS Rayleigh and LoS Rician fading for the network hops. The information theoretic work of [13] has also been considered an asymmetric scenario where the source's transmission experiences Rayleigh fading, while the relay transmission experiences a fixed amplitude gain additive white Gaussian noise (AWGN) channel.

In this work, the outage probability and the ABEP of a dual-hop relay system in an asymmetric fading environment is investigated. We assume that the source-relay and relaydestination links experience Rayleigh or Rician fading. First, using the cumulative distribution functions (cdfs) of the endto-end signal-to-noise ratio (SNR), the outage probability is derived. Next, using the cdf, the ABEP of $M$-ary square QAM modulation is derived. Simulation results are also presented to verify the theoretical analysis. 
The rest of the paper is organized as follows: In Section II we outline the system and channel model. In Section III, we derive outage probability expressions for the asymmetric channel. The ABEP of $M$-QAM modulated signals is computed in Section IV. Finally, we conclude with a brief summary of results in Section V.

\section{System AND Channel Model}

Assume a dual-hop fixed gain AF relay system (cf. [3, Fig. 1]) operating in an asymmetric fading environment. The source, $S$ communicates with the destination, $D$ using a relay, $R$. Each transmission period is divided into two signalling intervals: In the first signalling interval, the received signal at $R$ is multiplied by a gain factor $G$ and in the second signalling interval, it is retransmitted to $D$. Assuming that $S$ transmits a signal with an average power normalized to unity, the instantaneous end-to-end SNR at the destination, $\gamma_{\text {eq }}$, is [3]

$$
\gamma_{\mathrm{eq}}=\frac{\left(\alpha_{1}^{2} / N_{01}\right)\left(\alpha_{2}^{2} / N_{02}\right)}{\left(\alpha_{2}^{2} / N_{02}\right)+\left(1 / G^{2} N_{01}\right)}
$$

where $\alpha_{1}, \alpha_{2}$ are the fading amplitudes of the wireless channels in the $S-R$ and $R-D$ links respectively, $N_{01}$ and $N_{02}$ are the power of the AWGN component at the input of the relay and the destination, and $G$ is the relay gain. If $C=1 / G^{2} N_{01}$, $\gamma_{i}=\alpha_{i}^{2} / N_{0 i}$ for $i=1,2$ and then (1) simplifies to

$$
\gamma_{\mathrm{eq}}=\frac{\gamma_{1} \gamma_{2}}{C+\gamma_{2}} .
$$

In this work, we consider two cases for the fading distributions of the $S-R$ and $R-D$ links, namely:

- The $S-R$ link is subject to Rayleigh fading and the $R-D$ link is subject to Rician fading. In the following, this Rayleigh/Rician fading condition will be identified as scenario (a).

- The $S-R$ link is subject to Rician fading and the $R-$ $D$ link is subject to Rayleigh fading. In the following, this Rician/Rayleigh fading condition will be identified as scenario (b).

If a link experiences Rayleigh fading, $\gamma_{i}$, (with $i=1$ or 2 ), is an exponentially distributed random variable (RV). That is, its probability density function (pdf) is given by

$$
p_{\gamma_{i}}(\gamma)=\frac{1}{\bar{\gamma}_{i}} e^{-\gamma / \bar{\gamma}_{i}}
$$

where $\bar{\gamma}_{i}=\Omega_{i} / N_{0 i}$ and $\Omega_{i}$ is the average fading power of that link. by

If a link experiences Rician fading, the pdf of $\alpha_{i}$ is given

$$
p_{\alpha_{i}}(\alpha)=\frac{2(K+1) e^{-K} \alpha}{\Omega_{i}} e^{-\frac{(K+1) \alpha^{2}}{\Omega_{i}}} I_{0}\left(2 \alpha \sqrt{\frac{K(K+1)}{\Omega_{i}}}\right)
$$

where $K$ is the Rician $K$-factor defined as the ratio of the powers of the LoS component to the scattered components and $I_{0}(\cdot)$ is the zeroth order modified Bessel function of the first kind. $\gamma_{i}$ is distributed according to a noncentral- $\chi^{2}$ distribution given by

$$
p_{\gamma_{i}}(\gamma)=\frac{(K+1) e^{-K}}{\bar{\gamma}_{i}} e^{-\frac{(K+1) \gamma}{\bar{\gamma}_{i}}} I_{0}\left(2 \sqrt{\frac{K(K+1) \gamma}{\bar{\gamma}_{i}}}\right) .
$$

Observe that when $K=0$ the Rician distribution becomes the Rayleigh distribution. As $K \rightarrow \infty$, the distribution approximates that of an AWGN (no fading) channel. Values of the $K$-factor in indoor/outdoor land mobile applications normally range from $0-12 \mathrm{~dB}$ [8].

\section{OUtAge Probability}

Outage probability is an important performance measure that is commonly used to characterize a wireless communication system. It is defined as the probability that the instantaneous end-to-end SNR, $\gamma_{\text {eq }}$, falls below a threshold $\gamma_{\text {th }}$. Therefore mathematically, the outage probability is given by [3]

$$
P_{\text {out }}=F_{\gamma_{\mathrm{eq}}}\left(\gamma_{\mathrm{th}}\right)=\operatorname{Pr}\left[\frac{\gamma_{1} \gamma_{2}}{C+\gamma_{2}}<\gamma_{\mathrm{th}}\right]
$$

where $F_{\gamma_{\mathrm{eq}}}(\gamma)$ is the cdf of the end-to-end SNR. Next, we calculate the outage probability applicable to scenarios (a) and (b).

\section{A. Scenario (a)}

In the case of scenario (a) we can express $P_{\text {out }}$ as

$$
P_{\text {out }}=\int_{0}^{\infty} \operatorname{Pr}\left[\gamma_{1}<\frac{\gamma_{\text {th }}\left(C+\gamma_{2}\right)}{\gamma_{2}} \mid \gamma_{2}\right] p_{\gamma_{2}}\left(\gamma_{2}\right) d \gamma_{2} .
$$

The cdf of $\gamma_{1}$ is $1-e^{-\gamma / \bar{\gamma}_{1}}$, and using (5), (7) can be written as

$$
\begin{aligned}
P_{\text {out }} & =\frac{(K+1) e^{-K}}{\bar{\gamma}_{2}} \int_{0}^{\infty}\left[1-e^{-\left(\gamma_{\mathrm{th}} / \bar{\gamma}_{1}\right)\left(1+C / \gamma_{2}\right)}\right] \\
& \times e^{-\frac{(K+1) \gamma_{2}}{\bar{\gamma}_{2}}} I_{0}\left(2 \sqrt{\frac{K(K+1) \gamma_{2}}{\bar{\gamma}_{2}}}\right) d \gamma_{2} \\
& =1-\frac{(K+1) e^{-K}}{\bar{\gamma}_{2}} \int_{0}^{\infty} e^{-\left(\gamma_{\mathrm{th}} / \bar{\gamma}_{1}\right)\left(1+C / \gamma_{2}\right)} \\
& \times e^{-\frac{(K+1) \gamma_{2}}{\bar{\gamma}_{2}}} I_{0}\left(2 \sqrt{\frac{K(K+1) \gamma_{2}}{\bar{\gamma}_{2}}}\right) d \gamma_{2} .
\end{aligned}
$$

The integral required to compute the outage probability in (8) is

$$
I_{1}=\int_{0}^{\infty} e^{-C \gamma_{\mathrm{th}} /\left(\bar{\gamma}_{1} \gamma_{2}\right)-(K+1) \gamma_{2} / \bar{\gamma}_{2}} I_{0}\left(2 \sqrt{\frac{K(K+1) \gamma_{2}}{\bar{\gamma}_{2}}}\right) d \gamma_{2} .
$$

We are unaware of a closed-form analytical solution to this integral. Nevertheless, using the infinite-series representation of $I_{0}(x)$ [14, Eq. $\left.(8.447-1)\right]$

$$
I_{0}(x)=\sum_{\ell=0}^{\infty} \frac{x^{2 \ell}}{2^{2 \ell}(\ell !)^{2}}
$$


we can rewrite $I_{1}$ as

$$
I_{1}=\sum_{\ell=0}^{\infty} \frac{K^{\ell}(K+1)^{\ell}}{(\ell !)^{2} \bar{\gamma}_{2}^{\ell}} \int_{0}^{\infty} \gamma_{2}^{\ell} e^{-C \gamma_{\mathrm{th}} /\left(\bar{\gamma}_{1} \gamma_{2}\right)-(K+1) \gamma_{2} / \bar{\gamma}_{2}} d \gamma_{2}
$$

The integral in (11) can be evaluated using [14, Eq. (3.471-9)]. We write $I_{1}$ as

$$
\begin{aligned}
I_{1} & =\sum_{\ell=0}^{\infty} \frac{2 K^{\ell}}{(\ell !)^{2}}\left(\frac{K+1}{\bar{\gamma}_{2}}\right)^{\frac{\ell-1}{2}}\left(\frac{C \gamma_{\mathrm{th}}}{\bar{\gamma}_{1}}\right)^{\frac{\ell+1}{2}} \\
& \times \mathcal{K}_{\ell+1}\left(2 \sqrt{\frac{C(K+1) \gamma_{\mathrm{th}}}{\bar{\gamma}_{1} \bar{\gamma}_{2}}}\right)
\end{aligned}
$$

for $\gamma_{\text {th }}>0$ and $\mathcal{K}_{\nu}(\cdot)$ is the $\nu$ th-order modified Bessel function of the second kind. Finally, $P_{\text {out }}$, for scenario (a) is given by

$$
\begin{aligned}
P_{\text {out }} & =1-e^{-K-\gamma_{\mathrm{th}} / \bar{\gamma}_{1}} \sum_{\ell=0}^{\infty} \frac{2 K^{\ell}}{(\ell !)^{2}}\left(\frac{C(K+1) \gamma_{\mathrm{th}}}{\bar{\gamma}_{1} \bar{\gamma}_{2}}\right)^{\frac{\ell+1}{2}} \\
& \times \mathcal{K}_{\ell+1}\left(2 \sqrt{\frac{C(K+1) \gamma_{\mathrm{th}}}{\bar{\gamma}_{1} \bar{\gamma}_{2}}}\right) .
\end{aligned}
$$

Concerning the convergence of the infinite series in (13), the truncation error if $T_{1}$ terms are used is

$$
\begin{aligned}
R_{1} & =e^{-K-\gamma_{\mathrm{th}} / \bar{\gamma}_{1}} \sum_{\ell=T_{1}+1}^{\infty} \frac{2 K^{\ell}}{(\ell !)^{2}}\left(\frac{C(K+1) \gamma_{\mathrm{th}}}{\bar{\gamma}_{1} \bar{\gamma}_{2}}\right)^{\frac{\ell+1}{2}} \\
& \times \mathcal{K}_{\ell+1}\left(2 \sqrt{\frac{C(K+1) \gamma_{\mathrm{th}}}{\bar{\gamma}_{1} \bar{\gamma}_{2}}}\right)
\end{aligned}
$$

For $\nu>0$ and fixed $x, \mathcal{K}_{\nu}(x)$ can be asymptotically approximated as [15]

$$
K_{\nu}(x) \propto \frac{(\nu-1) !}{2}\left(\frac{x}{2}\right)^{-\nu}
$$

Substituting (15) in (14) and after simplifications we get

$$
R_{1} \propto e^{-K-\gamma_{\mathrm{th}} / \bar{\gamma}_{1}}\left(e^{K}-\sum_{\ell=0}^{T} \frac{K^{\ell}}{\ell !}\right)
$$

Finally using [16, Eq. (4.1.7.10)]

$$
R_{1} \propto e^{-\gamma_{\mathrm{th}} / \bar{\gamma}_{1}}\left(1-\frac{\Gamma\left(T_{1}+1, K\right)}{T_{1} !}\right)
$$

where $\Gamma(\alpha, x)=\int_{x}^{\infty} t^{\alpha-1} e^{-t} d t$ is the complementary incomplete gamma function [16, p. 792].

\section{B. Scenario (b)}

In the case of scenario (b) we can express $P_{\text {out }}$ as

$$
P_{\text {out }}=\int_{0}^{\infty} \operatorname{Pr}\left[\gamma_{2}<\frac{C \gamma_{\mathrm{th}}}{\left(\gamma_{1}-\gamma_{\mathrm{th}}\right)} \mid \gamma_{1}\right] p_{\gamma_{1}}\left(\gamma_{1}\right) d \gamma_{1}
$$

and after some manipulations (18) can be reexpressed as

$$
P_{\text {out }}=1-\int_{\gamma_{\text {th }}}^{\infty} e^{C \gamma_{\mathrm{th}} / \bar{\gamma}_{2}\left(\gamma_{1}-\gamma_{\mathrm{th}}\right)} p_{\gamma_{1}}\left(\gamma_{1}\right) d \gamma_{1}
$$

Using the Rician pdf of (5), the integral in (19) is given by

$$
\begin{aligned}
I_{2} & =\frac{(K+1) e^{-K}}{\bar{\gamma}_{1}} \int_{\gamma_{\mathrm{th}}}^{\infty} e^{C \gamma_{\mathrm{th}} / \bar{\gamma}_{2}\left(\gamma_{1}-\gamma_{\mathrm{th}}\right)} e^{-\frac{(K+1)}{\bar{\gamma}_{1}} \gamma_{1}} \\
& \times I_{0}\left(2 \sqrt{\frac{K(K+1) \gamma_{1}}{\bar{\gamma}_{1}}}\right) d \gamma_{1} .
\end{aligned}
$$

Let $u=\gamma_{1}-\gamma_{\mathrm{th}}$, then

$$
\begin{aligned}
I_{2} & =\frac{K+1}{\bar{\gamma}_{1}} e^{-K-\frac{(K+1) \gamma_{\mathrm{th}}}{\bar{\gamma}_{2}}} \int_{0}^{\infty} e^{-C \gamma_{\mathrm{th}} / \bar{\gamma}_{2} u} e^{-\frac{(K+1) u}{\bar{\gamma}_{1}}} \\
& \times I_{0}\left(2 \sqrt{\frac{K(K+1)\left(\gamma_{\mathrm{th}}+u\right)}{\bar{\gamma}_{1}}}\right) d u
\end{aligned}
$$

Invoking again the infinite series representation of $I_{0}(x)$ we can expand (21) as

$$
\begin{aligned}
I_{2} & =\frac{(K+1)}{\bar{\gamma}_{1}} e^{-K-\frac{(K+1) \gamma_{\text {th }}}{\bar{\gamma}_{1}}} \sum_{\ell=0}^{\infty} \frac{K^{\ell}(K+1)^{\ell}}{(\ell !)^{2} \bar{\gamma}_{1}^{\ell}} \\
& \times \int_{0}^{\infty} e^{-C \gamma_{\mathrm{th}} / \bar{\gamma}_{2} u} e^{-\frac{(K+1) u}{\bar{\gamma}_{1}}}\left(u+\gamma_{\mathrm{th}}\right)^{\ell} d u .
\end{aligned}
$$

Using the binomial expansion and the integral result of [14, Eq. (3.471-9)] $I_{2}$ can be solved. Therefore, $P_{\text {out }}$ for scenario (b) is expressed as

$$
\begin{aligned}
& P_{\text {out }}=1-e^{-K-\frac{(K+1) \gamma_{\text {th }}}{\bar{\gamma}_{1}}} \sum_{\ell=0}^{\infty} \frac{2 K^{\ell}}{(\ell !)^{2}} \sum_{r=0}^{\ell}\left(\begin{array}{l}
\ell \\
r
\end{array}\right) \gamma_{\text {th }}^{\frac{2 \ell-r+1}{2}} \\
& \times\left(\frac{K+1}{\bar{\gamma}_{1}}\right)^{\frac{2 \ell-r+1}{2}}\left(\frac{C}{\bar{\gamma}_{2}}\right)^{\frac{r+1}{2}} \mathcal{K}_{r+1}\left(2 \sqrt{\frac{C(K+1) \gamma_{\text {th }}}{\bar{\gamma}_{1} \bar{\gamma}_{2}}}\right) .
\end{aligned}
$$

For numerical verification, assume that both the $S-R$ and $R-D$ links experience Rayleigh fading, i.e., the scenario considered in [3]. Substituting $K=0$ in (13) and (23) we get [3, Eq. (9)].

In all cases of practical significance, the infinite series representations involved in (23) can be truncated without sacrificing numerical accuracy. Suppose that we truncate (23) after $T_{2}$ terms. Therefore, the remainder, $R_{2}$ after applying (15) becomes asymptotically

$$
\begin{aligned}
R_{2} & \propto e^{-K-\frac{(K+1) \gamma_{\mathrm{th}}}{\bar{\gamma}_{1}}} \sum_{\ell=T_{2}+1}^{\infty} \frac{1}{\ell !}\left(\frac{K(K+1) \gamma_{\mathrm{th}}}{\bar{\gamma}_{1}}\right)^{\ell} \\
& \times \sum_{r=0}^{\ell} \frac{1}{(\ell-r) !}\left(\frac{(K+1) \gamma_{\mathrm{th}}}{\bar{\gamma}_{1}}\right)^{-r}
\end{aligned}
$$

and simplifying further

$$
R_{2} \propto e^{-K} \sum_{\ell=T_{2}+1}^{\infty} \frac{K^{\ell}}{(\ell !)^{2}} \Gamma\left(\ell+1, \frac{(K+1) \gamma_{\mathrm{th}}}{\bar{\gamma}_{1}}\right) .
$$

We are unable to obtain a closed-form solution for the summation in (25). However note that, using the L'Hopital rule it can be shown that $\lim _{x \rightarrow \infty} K^{x} /(x !)^{2}=0$. Therefore, $R_{2}$ asymptotically converges to a finite number.

Figs. 1 and 2 show the outage probability for scenarios (a) and (b) respectively. In all cases, we have employed the fixed gain $G$ assumed in [4] under the so-called average power scaling. The theoretical results were plotted using the first 20 terms of the infinite series $(\ell=20)$, where they match exactly 


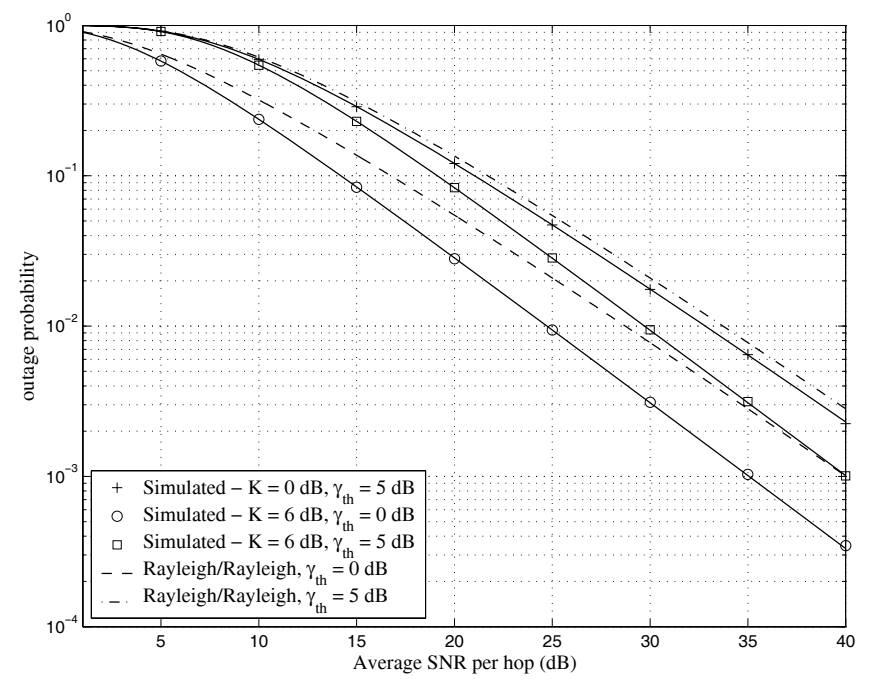

Fig. 1. Outage probability in Rayleigh/Rician fading.

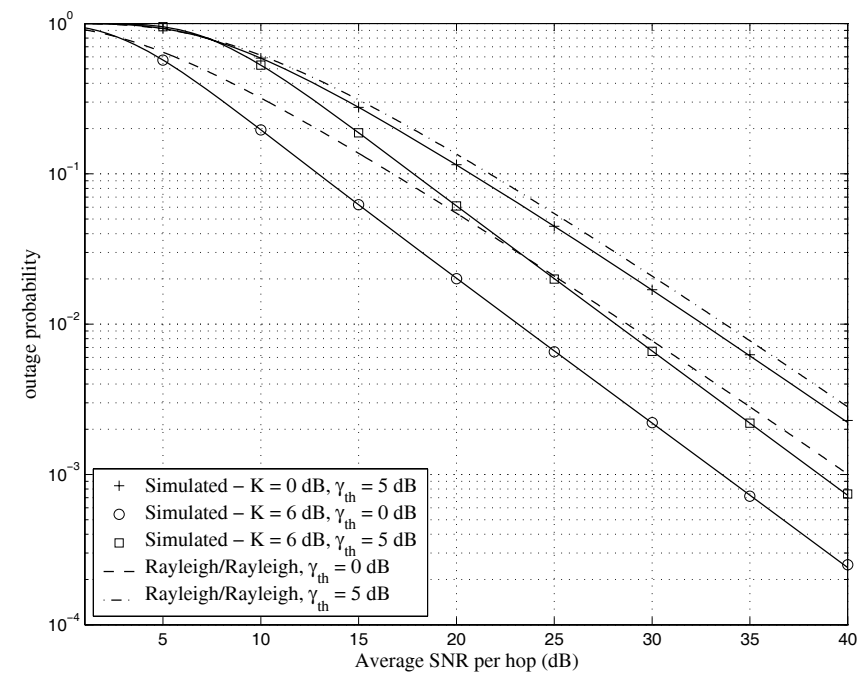

Fig. 2. Outage probability in Rician/Rayleigh fading.

with the simulated results. The plots in Figs. 1 and 2 show similar trends, i.e., with an increasing $K$-factor, outage events decrease. However, in all cases, the outage probability of Rician/Rayleigh fading is lower than Rayleigh/Rician fading. Better channel conditions in the first hop (Rician fading) leads to an outage performance improvement because the end-to-end SNR probabilistically reaches higher values more often than in the first hop Rayleigh fading scenario.

\section{Average Bit Error Probability}

In this section we evaluate the ABEP of the dual-hop relay network assuming that the transmitted data are modulated using a $M$-ary square QAM alphabet which transmits data by changing the amplitude of two carrier signals, and is widely used in wireless communication systems.

The ABEP is a useful measure of evaluating the performance of wireless communication applications. Traditionally the ABEP is computed by determining the pdf of $\gamma_{\text {eq }}$ and then averaging the conditional BEP in AWGN, $P_{b}(e \mid \gamma)$, over this pdf. Mathematically, $P_{b}(e)$ in the relay channel is given by

$$
P_{b}(e)=\int_{0}^{\infty} P_{b}(e \mid \gamma) p_{\gamma_{\mathrm{eq}}}(\gamma) d \gamma
$$

Using the BEP in an AWGN channel [17, Eq. (14)], the $k$-th bit ABEP of Gray bit-mapped $M$-ary square QAM is given by

$$
\begin{aligned}
& P_{b}(e \mid k)=\frac{2}{\sqrt{M}} \sum_{i=0}^{\left(1-2^{-k}\right) \sqrt{M}-1}\left\{(-1)^{\left\lfloor\frac{i .2^{k-1}}{\sqrt{M}}\right\rfloor}\right. \\
& \left.\times\left(2^{k-1}-\left\lfloor\frac{i .2^{k-1}}{\sqrt{M}}+\frac{1}{2}\right\rfloor\right) \mathcal{E}\left[Q\left((2 i+1) \sqrt{\frac{3 \gamma}{M-1}}\right)\right]\right\}
\end{aligned}
$$

where $\mathcal{E}[\cdot]$ denotes the statistical expectation operator, $Q(x)$ being the Gaussian $Q$-function defined as $Q(x)=$ $(1 / \sqrt{2 \pi}) \int_{x}^{\infty} e^{-t^{2} / 2} d t$ and $\lfloor x\rfloor$ is the largest integer smaller or equal to $x$. Finally, the ABEP of $M$-ary square QAM is calculated from,

$$
P_{b}(e)=\frac{1}{\log _{2} \sqrt{M}} \sum_{k=0}^{\log _{2} \sqrt{M}} P_{b}(e \mid k)
$$

In order to compute the ABEP for scenarios (a) and (b) we identify that the integral that needs to be computed is of the form:

$$
\mathcal{J}=\int_{0}^{\infty} Q(\sqrt{\beta \gamma}) p_{\gamma_{\text {eq }}}(\gamma) d \gamma
$$

where $\beta$ is a constant. Note, that $\mathcal{J}$ can be computed using the method presented in [7]. After integration by parts and the change of variable $t=\sqrt{\beta \gamma}$, (29) can be rewritten as

$$
\mathcal{J}=\frac{1}{\sqrt{2 \pi}} \int_{0}^{\infty} F_{\gamma_{\text {eq }}}\left(\frac{t^{2}}{\beta}\right) e^{-\frac{t^{2}}{2}} d t
$$

Consider scenario (a): Using (30), $\mathcal{J}$, can be written as

$$
\begin{aligned}
\mathcal{J}_{1} & =\frac{1}{2}-\frac{e^{-K}}{\sqrt{2 \pi}} \sum_{\ell=0}^{\infty} \frac{2 K^{\ell}}{(\ell !)^{2}}\left(\frac{C(K+1)}{\bar{\gamma}_{1} \bar{\gamma}_{2} \beta}\right)^{\frac{\ell+1}{2}} \\
& \times \int_{0}^{\infty} t^{\ell+1} e^{-\left(\frac{2+\bar{\gamma}_{1} \beta}{2 \bar{\gamma}_{1} \beta}\right) t^{2}} \mathcal{K}_{\ell+1}\left(2 \sqrt{\frac{C(K+1)}{\bar{\gamma}_{1} \bar{\gamma}_{2} \beta}} t\right) d t .
\end{aligned}
$$

Using [14, Eq. (6.631-3)], (31) can be evaluated as

$$
\begin{aligned}
& \mathcal{J}_{1}=\frac{1}{2}-e^{-K} \sum_{\ell=0}^{\infty} \frac{(2 K)^{\ell}}{(\ell !)^{2} \sqrt{1+2 /\left(\bar{\gamma}_{1} \beta\right)}} \Gamma\left(\ell+\frac{3}{2}\right) \\
& \times\left(\frac{C(K+1)}{\left(2+\bar{\gamma}_{1} \beta\right) \bar{\gamma}_{2}}\right)^{\ell+1} \Psi\left(\ell+\frac{3}{2}, \ell+2 ; \frac{2 C(K+1)}{\left(2+\bar{\gamma}_{1} \beta\right) \bar{\gamma}_{2}}\right)
\end{aligned}
$$

where $\Psi(a, b ; z)$ is the Tricomi function (also known as the Kummer $U$ function) [18], defined as $\Psi(a, c ; z)=$ $\frac{1}{\Gamma(a)} \int_{0}^{\infty} e^{-z t} t^{a-1}(1+t)^{c-a-1} d t[19$, p. 504] and $\Gamma(x)=$ $\int_{0}^{\infty} t^{z-1} e^{-t} d t$ is the gamma function. We have employed the well known relationship between the Whittaker function and $\Psi(a, b ; z)[19$, p. 505] to arrive at (32). Note, that $\Psi(\cdot, \cdot ; z)$ can be evaluated using popular symbolic software such as MATLAB, MAPLE and MATHEMATICA. Finally, after substituting (32) with $\beta=3(2 i+1)^{2} /(M-1)$ in (27) and using (28) we arrive at the ABEP. 
Tricomi function $\Psi(a, b, z)$ is a monotonically decreasing function respect to $a$ and $b$. This means that when $a$ and $b$ increase Tricomi decreases. Therefore, the truncation error, $R_{3}$ in (32) after $T_{3}$ terms can be upper bounded as

$$
\begin{aligned}
R_{3} & <\Psi\left(T_{3}+\frac{5}{2}, T_{3}+3 ; \frac{2 C(K+1)}{\left(2+\bar{\gamma}_{1} \beta\right) \bar{\gamma}_{2}}\right) e^{-K} \\
& \times \sum_{\ell=T_{3}+1}^{\infty} \frac{(2 K)^{\ell} \Gamma\left(\ell+\frac{3}{2}\right)}{(\ell !)^{2} \sqrt{1+2 /\left(\bar{\gamma}_{1} \beta\right)}}\left(\frac{C(K+1)}{\left(2+\bar{\gamma}_{1} \beta\right) \bar{\gamma}_{2}}\right)^{\ell+1} .
\end{aligned}
$$

Using the identity $\Gamma\left(\ell+\frac{3}{2}\right)=\frac{\sqrt{\pi}(2 \ell+1) !}{2^{2 \ell+1} \ell !}[16$, p. 773] and expressing the summation of (33) in closed-form we finally obtain

$$
\begin{aligned}
& R_{3}<\frac{(2 K)^{T_{3}+1} \Gamma\left(T_{3}+\frac{5}{2}\right)}{\left(\left(T_{3}+1\right) !\right)^{2} \sqrt{1+2 /\left(\bar{\gamma}_{1} \beta\right)}}\left(\frac{C(K+1)}{\left(2+\bar{\gamma}_{1} \beta\right) \bar{\gamma}_{2}}\right)^{T_{3}+2} \\
& \times e^{-K} \Psi\left(T_{3}+\frac{5}{2}, T_{3}+3 ; \frac{2 C(K+1)}{\left(2+\bar{\gamma}_{1} \beta\right) \bar{\gamma}_{2}}\right) \\
& \times{ }_{2} F_{2}\left(1, T_{3}+\frac{5}{2} ; T_{3}+2, T_{3}+2 ; \frac{2 C K(K+1)}{\left(2+\bar{\gamma}_{1} \beta\right) \bar{\gamma}_{2}}\right)
\end{aligned}
$$

where ${ }_{p} F_{q}\left(a_{1}, \ldots, a_{p} ; b_{1}, \ldots, b_{q} ; z\right)$ is the generalized hypergeometric function [16, p. 788].

Consider scenario (b). Substituting $F_{\gamma_{\mathrm{eq}}}(\gamma)$ of (23) in (30) we obtain

$$
\begin{aligned}
\mathcal{J}_{2} & =\frac{1}{2}-\frac{e^{-K}}{\sqrt{\pi}} \sum_{\ell=0}^{\infty} \frac{\sqrt{2}(K)^{\ell}}{(\ell !)^{2}} \sum_{r=0}^{\ell}\left(\begin{array}{l}
\ell \\
r
\end{array}\right) \\
& \times\left(\frac{K+1}{\bar{\gamma}_{1}}\right)^{\frac{2 \ell-r-1}{2}}\left(\frac{C}{\bar{\gamma}_{2}}\right)^{\frac{r+1}{2}} \beta^{\frac{r-2 \ell-1}{2}} \int_{0}^{\infty} t^{2 \ell-r+1} \\
& \times e^{-\left(\frac{2(K+1)+\bar{\gamma}_{1} \beta}{2 \bar{\gamma}_{1} \beta}\right) t^{2}} \mathcal{K}_{r+1}\left(2 \sqrt{\frac{C(K+1)}{\bar{\gamma}_{1} \bar{\gamma}_{2} \beta}} t\right) d t
\end{aligned}
$$

Using [14, Eq. (6.631-3)], $\mathcal{J}_{2}$ can be simplified as shown at the top of the next page.

Note that by exploiting the monotonic behavior of the Tricomi function as in (33) and using the L'Hopital rule, we can show that (36) converge. This proof is omitted here due to limited space.

Figs. 3 and 4 show the ABEP performance of the dualhop relay network over Rayleigh/Rician and Rician/Rayleigh fading. All cases correspond to 4-QAM and 16-QAM. Theoretical results were obtained by truncating the infinite series of (32) and (36) to 20 terms, i.e., $\ell=20$. The number of terms required in (32) and (36) to achieve a given figure accuracy depend on the SNR and the $K$-factor. For example, to achieve six significant figure accuracy at $K=0 \mathrm{~dB}$, only eight terms are necessary, while at $K=20 \mathrm{~dB}, 250$ terms are required. However compared to time consuming computer simulations, in all cases, the computational load required for calculating the theoretical ABEP is marginal.

In Fig. 3 we have also plotted the performance curves for Rayleigh/Rayleigh fading for comparison purposes. Under Rayleigh/Rayleigh fading, $\mathcal{J}$ simplifies to

$$
\mathcal{J}=\frac{1}{2}\left(1-\frac{\varphi\left(K_{1}(\varphi)-K_{0}(\varphi)\right) e^{\varphi}}{\sqrt{1+\left(2 / \bar{\gamma}_{1} \beta\right)}}\right),
$$

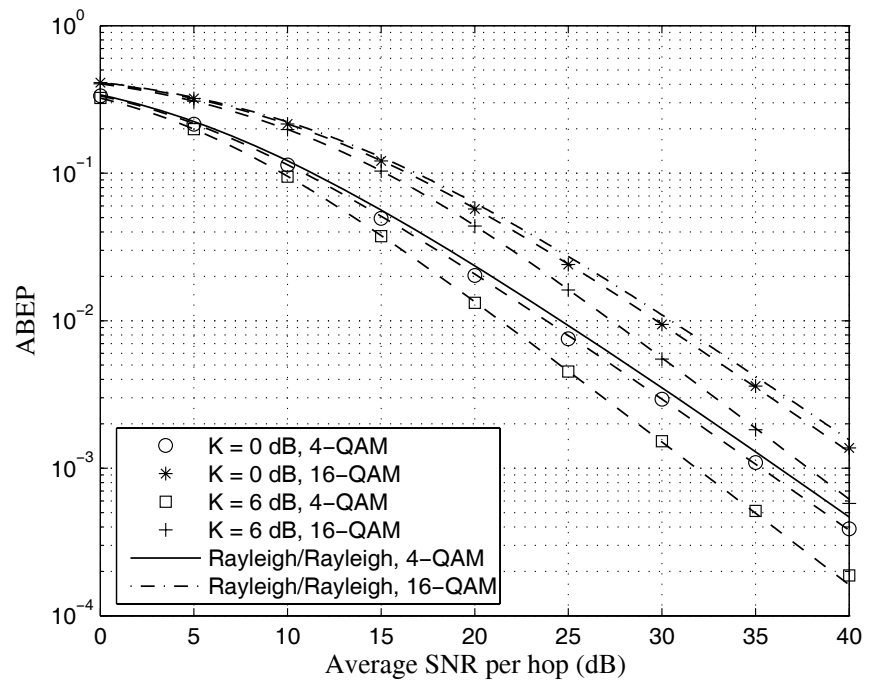

Fig. 3. ABEP of 4 and 16-QAM in Rayleigh/Rician fading. Dashed lines and "markers" denote theoretical and simulated ABEPs respectively.

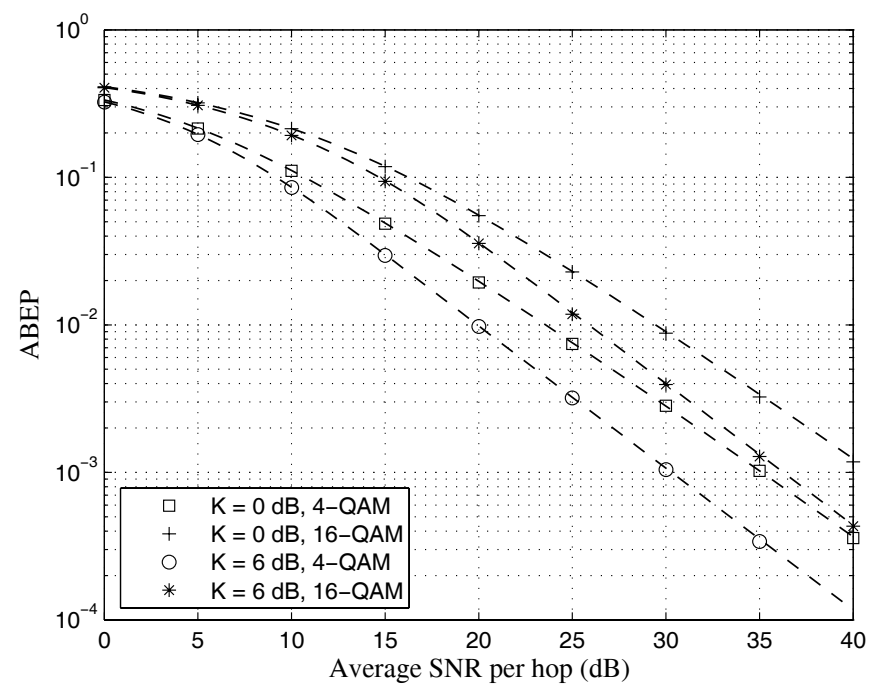

Fig. 4. ABEP of 4 and 16-QAM in Rician/Rayleigh fading. Dashed lines and "markers" denote theoretical and simulated ABEPs respectively.

where $\varphi=C /\left(2+\bar{\gamma}_{1} \beta\right) \bar{\gamma}_{2}$. Clearly, Rayleigh/Rayleigh fading deteriorates the ABEP of the relay system compared to both asymmetric fading conditions. In all cases, theoretical ABEPs and simulations match very well. For a small $K$-factor $(0 \mathrm{~dB})$, Figs. 3 and 4 illustrate that ABEP of scenarios (a) and (b) are almost identical. However, for a typical $K$-factor $(6 \mathrm{~dB})$ and at high SNR, Rician/Rayleigh fading is able to provide a slightly better error performance compared to Rayleigh/Rician fading. This is due to the fact in AF systems with fixed-gain, the endto-end performance is dominated by the first hop channel gain. This is evident from (2). Therefore, better channel conditions in the first hop (Rician fading) leads to improved performance compared to the first hop Rayleigh fading scenario.

\section{CONCLUSIONS}

In this paper, we have presented two infinite-series representations for the outage probability of a dual-hop communication system equipped with a single fixed-gain amplifyand-forward relay in Rayleigh/Rician and Rician/Rayleigh 


$$
\begin{aligned}
\mathcal{J}_{2}=\frac{1}{2}-\frac{e^{-K}}{\sqrt{\pi\left(1+2(K+1) /\left(\bar{\gamma}_{1} \beta\right)\right)}} & \sum_{\ell=0}^{\infty} \frac{(2 K)^{\ell}}{(\ell !)^{2}\left(2+\bar{\gamma}_{1} \beta /(K+1)\right)^{\ell+1}} \Gamma\left(\ell+\frac{3}{2}\right) \\
& \sum_{r=0}^{\ell}\left(\begin{array}{l}
\ell \\
r
\end{array}\right)\left(\frac{C}{\bar{\gamma}_{2}}\right)^{r+1} \Gamma\left(\ell-r+\frac{1}{2}\right) \Psi\left(\ell+\frac{3}{2}, r+2 ; \frac{2 C(K+1)}{\left(2(K+1)+\bar{\gamma}_{1} \beta\right) \bar{\gamma}_{2}}\right) .
\end{aligned}
$$

fading environments respectively. Based on these expressions, the average bit error probability (ABEP) of $M$-ary square QAM was also derived. These results demonstrate that the system exhibits an improved performance in a Rician/Rayleigh (source-relay link/relay-destination link) environment compared to a Rayleigh/Rician environment. Furthermore, both Rayleigh/Rician and Rician/Rayleigh asymmetric fading environments, compared to the scenario where both links suffer only from Rayleigh fading (Rayleigh/Rayleigh), produce improved ABEPs. Simulation results for the outage probability and the ABEP are in excellent agreement with the theoretical results obtained in this paper. Since it has been found in practice that different links of a relay system could experience line-of-sight/non line-of-sight fading conditions, this analysis is useful to the system design engineer for performance evaluation purposes.

\section{REFERENCES}

[1] R. Pabst, B. H. Walke, D. C. Schultz, et al., "Relay-based deployment concepts for wireless and mobile broadband radio," IEEE Commun. Mag., vol. 42, pp. 80-89, Sept. 2004.

[2] Y. Fan and J. Thompson, "MIMO configurations for relay channels: theory and practice," IEEE Trans. Wireless Commun., vol. 6, pp. 17741786, May 2007.

[3] M. O. Hasna and M.-S. Alouini, "A performance study of dual-hop transmissions with fixed gain relays," IEEE Trans. Wireless Commun., vol. 3, pp. 1963-1968, Nov. 2004.

[4] H. Mheidat and M. Uysal, "Impact of receive diversity on the performance of amplify-and-forward relaying under APS and IPS power constraints," IEEE Commun. Lett., vol. 10, pp. 468-470, June 2006.

[5] G. K. Karagiannidis, T. A. Tsiftsis, and R. K. Mallik, "Bounds of multihop relayed communications in Nakagami- $m$ fading," IEEE Trans. Commun., vol. 54, pp. 18-22, Jan. 2006.
[6] G. K. Karagiannidis, "Performance bounds of multihop wireless communications with blind relays over generalized fading channels," IEEE Trans. Wireless Commun., vol. 5, pp. 498-503, Mar. 2006.

[7] Y. Zhao and R. Adve, "Symbol error rate of selection amplify-andforward relay systems," IEEE Commun. Lett., pp. 757-759, Nov. 2006.

[8] J. D. Parsons, The Mobile Radio Propagation Channel. New York: Wiley, 1992.

[9] P. Kyösti, J. Meinilä, L. Hentilä, et al., WINNER II Interim Channel Models (IST-4-027756 WINNER II D1.1.1 V1.1) [Online] Available: http://www.ist-winner.org/WINNER2-Deliverables/D1.1.1.pdf.

[10] J. Adeane, M. R. D. Rodrigues, and I. J. Wassell, "Characterisation of the performance of cooperative networks in Rician fading channels," in Proc. 12th Intl. Conf. Telecommun. (ICT 2005), Cape Town, South Africa, May 2005.

[11] T. Q. Doung, H. Shin, and E.-K. Hong, "Effect of line-of-sight on dualhop nonregenerative relay wireless communications," in Proc. IEEE Vehicular Technol. Conf. (VTC 2007 Fall), Baltimore, MD, Sept./Oct. 2007, pp. 571-575.

[12] A. Adinoyi and H. Yanikomeroglu, "On the performance of cooperative wireless fixed relays in asymmetric channels," in Proc. IEEE GLOBECOM 2006, San Franscisco, CA, Nov./Dec. 2006, pp. 1-5.

[13] M. Katz and S. Shamai (Shitz), "Relaying protocols for two colocated users," IEEE Trans. Inform. Theory, vol. 52, pp. 2329-2344, June 2006.

[14] I. S. Gradshteyn and I. M. Ryzhik, Table of Integrals, Series and Products, 6th ed. San Diego: CA, Academic Press, 2000.

[15] R. Penfold, J.-M. Vanden-Broeck, and S. Grandison, "Monotonicity of some modified Bessel function products," Integral Transforms and Special Functions, vol. 18, no. 2, pp. 139-144, Feb. 2007.

[16] A. P. Prudnikov, Y. A. Brychkov, and O. I. Marichev, Integrals and Series, vol. 1. New York: Gordon and Breach Science Publishes, 1986.

[17] K. Cho and D. Yoon, "On the general BER expression of one and two dimensional amplitude modulations," IEEE Trans. Commun., vol. 50, pp. 1074-1080, July 2002.

[18] I. C. Sikaneta, "Numerically computing the Kummer $U$ function and a special case of the Gauss hypergeometric function," Defence R\&D Canada Technical Report (TR 2006-294), Dec. 2006. [Online] Available: http://www.ottawa.drdc-rddc.gc.ca/html/tr_2006_294_e.html.

[19] M. Abramowitz and I. A. Stegun, Handbook of Mathematical Functions with Formulas, Graphs, and Mathematical Tables, 10th ed. New York: Dover, 1972. 